\title{
Chronic obstructive pulmonary disease (COPD) and erectile dysfunction (ED): Results of the BRED observational study
}

\author{
Stefano Lauretti ${ }^{1}$, Vittorio Cardaci ${ }^{2}$, Francesco Barrese ${ }^{3}$, Luigino Calzetta ${ }^{4}$ \\ ${ }^{1}$ Center of Sexual Medicine and Urological Rehabilitation. Day Surgery Unit, PSCR S. Caterina della Rosa, ASL Roma C, Italy; \\ ${ }^{2}$ Physiopathology and Respiratory Rehabilitation Unit IRCSS San Raffaele Pisana, Rome, Italy; \\ ${ }^{3}$ Unit of Urology, Fabia Mater, Rome, Consultant in Urology IRCSS San Raffaele, Rome, Italy; \\ ${ }^{4}$ Laboratory of Systems Approaches, IRCSS San Raffaele Pisana, Rome, Italy.
}

\begin{abstract}
Summary
Most patients with chronic obstructive pulmonary disease (COPD) share many risk factors and similar aetiological agents with erectile dysfunction (ED). Both conditions also cause serious interference with quality of life and sexual relationships. In general, ageing and chronic illness decrease sexual interest, sexual function, and testosterone levels. This observational study included 66 male patients referred to our centre with different grades of COPD. We studied the different correlations between COPD and ED. The data collected from each patient regarded the following features: demographic and social condition; smoking status; clinical status; spirometric measurements. In this group, COPD was diagnosed in $78.8 \%$ and ED was present in $83.3 \%$ with increased severity in presence of LUTS and nicotinism.
\end{abstract}

KEY WORDS: Chronic obstructive pulmonary disease (COPD); Erectile dysfunction; Endothelial dysfunction, Chronic inflammation.

Submitted 18 August 2015; Accepted 13 October 2015

\section{INTRODUCTION}

Chronic obstructive pulmonary disease (COPD) is the persistent obstruction of airways (bronchiolitis) associated with the usually progressive destruction of lung areas (emphysema) associated with medical history of chronic inflammation, related to exposure to various pathogens including the prominent role attributed to cigarette smoking. Clinically it occurs with progressive shortness of breath, at first under stress and later - in severe cases even at rest, cough and chronic catarrh (1).

Erectile dysfunction (ED), or the "persistent inability to achieve and maintain an erection sufficient to permit satisfactory sexual intercourse" (2), not only implies a more or less severe deficit from a male biological functional point of view, but has also a particularly significant impact on relationships and the quality life of the man who is affected as well as on his partner. Currently epidemiological data still comply with the incidence values reported in the MMAS study, between 5 and 20\% of men, with mild to severe medical history (3).
ED shares several risk factors (4-5) with hypertension (two-fold risk), chronic cardiovascular disease (four-fold risk) (6), diabetes (three-fold risk) (7) and the resulting predisposing factors such as hypercholesterolemia (8), obesity as well as a sedentary lifestyle with contextual nicotinism, irrespective of the abuse entity, further increase the weight of the cause/effect relationship.

In a consistently stable fashion, wider epidemiological studies reveal how men between 50 and 59 years are the most frequently represented category, while below 40 years of age, the prevalence of the disorder is between $1 \%$ and $10 \%$, with an increase between 2 and $9 \%$ in the following decade, with a maximum of $15 \%$, then up to $50-100 \%$, from 70 years onwards. The association with various chronic conditions confirms an overall average increase of prevalence, which in the relationship between ED and diabetes, for example, is between 25 and $33 \%$, with peaks up to $77 \%$ (9-10-11).

Other predominantly chronic conditions may be associated with erectile disorder both in terms of cause and maintenance: renal or hepatic impairment; endocrinopathies such as hypogonadism, hypothyroidism and hyperprolactinemia; various neurological diseases such as postischemic outcomes, multiple sclerosis or Parkinson's disease and psychiatric disorders with particular regard to anxious and depressive syndromes (12-13).

More recently, increasing importance has been attributed to the common association with obesity and metabolic syndrome (14), although it is not yet clear whether these two conditions are to be understood as a separate entity or an additional risk to diabetes or cardiovascular diseases and Lower Urinary Tract Symptoms (LUTS) (15), which in the results of the Cologne Male Survey correlate with ED at a rate of $72 \%$ (16).

COPD and ED are two conditions that apparently have little in common. Certainly it is virtually impossible to compare their consequences on overall health and longterm prognosis (quod vitam), the therapeutic commitment in drug, instrument, rehabilitation and - consequently - economical terms, inevitably involves the entire family sphere. In the face of all this, however, both of these conditions not only present numerous patho- 
physiological mechanisms in common, and hence the possibility of shared approach. Furthermore, if we consider the population suffering from respiratory disease, what emerges is the constant demand for support in order to live a rewarding relationship and gratifying sexuality, which are extremely important in the early phases of the disease. In chronic disabling diseases, in fact, the sexual function can be otherwise altered, with negative consequences on the relationship, and more generally on the so-called quality of life (17-18). In men whose lives are often strongly affected by the disabling effects of chronic disease, sexuality can be a crucial moment of intimacy and a powerful source of pleasure, as well as of self-esteem and gratification and can help revive a positive affirmation of their role with their partner, whereas, in other contexts, such as work, sport, the social life, this role is limited or may have failed (19-21).

COPD is not a single nosological entity, but is rather a definition that gathers and describes chronic pulmonary conditions that cause a more or less important limitation of respiratory flow (22). Thus ED recognizes numerous aetiopathogenetic factors in common with chronic illnesses and, overall, an increased risk of incidence as has clearly emerged from various epidemiological investigations (23).

\section{Materials AND METHOdS}

At the Unit of Pathophysiology and Respiratory Rehabilitation at the IRCSS San Raffaele in Rome, an observational study on Broncopathy and Erectile Dysfunction -BRED was conducted from February 1 to July 30, 2015. 66 patients were studied in order to study the different correlations between COPD and ED. A database was designed and implemented in Visual Basic for Excel: data were then statistically evaluated using SPSS/v. 13.

Information gathered from each patient concerns the following features:

- Demographic and social: reason for visit, age, place of residence, marital status, type of work involving exposure or non-exposure to risk of respiratory diseases, perceived quality of life (St. George's scale);

- Smoking habits: active smoker, number of cigarettes smoked daily, years as smoker or ex-smoker;

- Clinical: co-morbidity/comorbidities, body mass index (BMI), blood glucose, total cholesterol, dyspnoea (MRC), COPD, erectile function (International Index of Erectile Function, IIEF), enlarged prostate (International Index symptoms of prostate, IPSS);

- Spirometric measurements: $\mathrm{pH}, \mathrm{CO}_{2}, \mathrm{O}_{2}$, Forced expiratory volume (FEV1), forced total capacity (FVC), Tiffenau Index (TI), forced expiatory flow FEF75\%, PEF, vital capacity (VC), total lung capacity (TLC). The reference ranges are summarized in Table 1.

COPD is expressed as a dichotomous categorical variable, while ED is also expressed as continuous. It should be specified that the latter is one of several fields of sexuality under investigation by the IIEF. The score obtained from particular applications allows to classify ED as shown in Table 2.

The distribution of absolute frequencies and percentages
Table 1.

Blood gas analysis and spirometric parameters and relative normality ranges.

\begin{tabular}{|lc|}
\hline Parameters & Normality ranges \\
\hline $\mathrm{PH}$ & $7.35-7.45$ \\
\hline $\mathrm{CO}_{2}$ & $35 \%-45 \%$ \\
\hline $\mathrm{O}_{2}$ & $>70 \%$ \\
\hline $\mathrm{FEV}_{1}$ & $>80 \%$ \\
\hline $\mathrm{FVC}$ & $>80 \%$ \\
\hline $\mathrm{IT}$ & $>80 \%$ \\
\hline $\mathrm{FEF}$ & $>80 \%$ \\
\hline $\mathrm{PEF}$ & $>80 \%$ \\
\hline $\mathrm{CV}$ & $>80 \%$ \\
\hline $\mathrm{TLC}$ & $<100 \%$ \\
\hline
\end{tabular}

Table 2.

Classification of ED.

\begin{tabular}{|lc|}
\hline Score & Dysfunction \\
\hline $1-10$ & Severe \\
\hline $11-16$ & Moderate \\
\hline $17-25$ & Light \\
\hline $26-30$ & Absent \\
\hline
\end{tabular}

of categorical variables and descriptive statistics for continuous variables were used to summarize the characteristics of the sample as a whole, according to the severity of ED and COPD. To assess differences in the various characteristics of patients in the different groups, the Chi-square test (or Fisher's exact test) for categorical explanatory variables and non-parametric tests were used, given the non-normality of the data in question for the constant explanatory variables.

In particular, Kruskal-Wallis allowed the comparison of the various degrees of severity of the disease, while the Mann-Whitney test assessed the presence or absence of diagnosis of the disease. Both tests assess the function of divers variables.

These variables were further explored through Spearman nonparametric correlation coefficients, to identify possible relationships with the diagnosis and severity of ED and COPD. Correlations were calculated first for the entire sample and subsequently for the stratified sample according to the following category variables: active smoker, years smoked, years as former smoker, number of cigarettes per day, comorbidity/co-morbidities, BMI, dyspnoea.

Ultimately a logistic regression model was applied in order to identify possible predictors of ED and COPD. Critical limit for significance was set at $5 \%$. The software used for the data analysis is SPSS/v 13.0.

\section{RESULTS}

The sample from 39 to 72 year-olds, had a mean age of $62.4 \pm 6.9$. ED was present in 55 subjects (83.3\%): 32 were severe (48.5\%), 6 moderate (9.1\%), 17 light (25.8\%) 
and the remaining 11 (16.7\%) had a negative diagnosis. $40.9 \%(n=27)$ of the patients had undergone medical examination for dyspnoea, 45.5\% $(n=30)$, for cough, $12.1 \%(n=8)$ for causes related to erectile functionality, one patient (1.5\%) for a generic check up.

COPD was diagnosed in 52 patients (78.8\%), among whom 10 (15.2\%) had slight COPD, 19 (28.8\%) moderate, $13(19.7 \%)$ severe, $10(15.2 \%)$ very serious and the remaining $14(21.2 \%)$ were at risk. $13.6 \%(n=9)$ of the patients had hypertension, $19.7 \%(\mathrm{n}=13)$, hypercholesterolemia, 15.2\% ( $\mathrm{n}=10)$ suffered from several other diseases, and the remaining $51.5 \%$ did not have co-morbidities.

90.9\% ( $\mathrm{n}=60)$ of the patients lived in cities while the rest of them lived in the country; 7 (10.6\%) had a job that exposed them to lung disease risks; $56.1 \%(\mathrm{n}=37)$ were married or cohabiting, $31.8 \%(n=21)$, were celibate, the remaining $12.2 \%$ were separated $(n=4)$ or widowed $(n=4)$.

According to the St. Georges questionnaire it was found that these patients perceived a low quality life $(86.4 \pm$ 19.3). The mean sample of patients had $28.3 \pm 6.7 \mathrm{BMI}$, 2 were underweight $(3.0 \%$ ) while 3 suffered severe obesity $(4.5 \%)$.

Taking into account the smoking habits, $21.2 \%(n=14)$ had never smoked, 24.2\% ( $\mathrm{n}=16)$ were smokers and the remaining $54.5 \%(n=36)$ had a history of smoking, including 30.6\% ( $\mathrm{n}=11)$ who had stopped smoking for less than a year, and $16.7 \%(\mathrm{n}=6)$ for over five years.

Considering the sample as a whole, $12.1 \%(\mathrm{n}=8)$ smoked for less than ten years, and 28.8\% $(n=19)$ for more than twenty years. Only one subject (1.5\%) smoked less than 5 cigarettes a day, one-third of the remaining (30.8\%) smoked between five and ten cigarettes a day, another third (34.6\%) between ten and twenty, while the last third $(32.7 \%)$ over twenty cigarettes per day.

24.2\% ( $\mathrm{n}=16)$ had no dyspnoea, 39.4\% ( $\mathrm{n}=26)$ light dyspnoea, 18.2\% ( $n=12)$ moderate, $16.7 \%(n=11)$ severe and only one case (1.5\%) suffered from extremely severe dyspnoea.

According to the IPSS scale, in the cases that showed lower urinary tract disorders, 39.4\% ( $n=26)$ of the cases had light symptoms, $10.6 \%(\mathrm{n}=7)$ of patients were seriously affected, while $50.0 \%(n=33)$ were seriously compromised only under certain circumstances.

With regard to the spirometric parameters, $87.9 \%$ ( $\mathrm{n}=$ 58) of the patients had normal values of $\mathrm{pH}, 3 \%(\mathrm{n}=2)$ lower than normal and 9.1\% $(n=6)$ higher than the threshold of normality (average $=7.4 ; \mathrm{SD}=0.0$ ).

Half of the patients had CO2 levels above the normal range, while none had lower values (average $=47.8 ; \mathrm{SD}$ $=12.9$ ).

62.1\% ( $\mathrm{n}=41)$ had normal oxygen levels while the remaining $37.9 \%(n=25)$ had low levels (average $=$ 73.8; ds 11.3).

Almost $70 \%(n=46)$ of patients had low FVC, the remaining 30\% ( $\mathrm{n}=20$ ) had normal (average $=69.4 ; \mathrm{SD}$ $=22.6)$.

$84.8 \%(n=56)$ were found to have too low FEV1 values, while the remaining 15.2\% ( $\mathrm{n}=10)$ had normal (average $=53.5 ; \mathrm{SD}=26.9)$.

Only $12.1 \%(\mathrm{n}=8)$ fulfilled the Tiffenau Index normali- ty parameter, against $87.9 \%(\mathrm{n}=58)$ who were below the threshold (average $=57.7 ; \mathrm{SD}=21.3$ ).

Slightly fewer than 30\% ( $=19)$ had a normal vital capacity, compared with $71.2 \%(n=47)($ mean $=70.6$; $\mathrm{SD}=21.7$ ).

Most patients, 51 (77.3\%), had a total lung capacity that was higher than $100 \%$, while only $22.7 \%(\mathrm{n}=15)$ was below the threshold (average $=145.4 ; \mathrm{SD}=64.5$ ).

Studying the distribution of the subjects characteristics related to the severity degrees of ED and COPD, it was found that: $56.8 \%(n=21)$ of married or cohabiting patients had severe ED, while the single men were affected by the disease only in $37.9 \%(\mathrm{n}=11)$ of the cases and in $41.4 \%(\mathrm{n}=12)$, the ED was light $\left(\mathrm{X}^{2}(3)=\right.$ $7.9, p=0.048) ;-42.3 \%(n=11)$ of the patients with absence or light urinary tract disorder suffered from light $\mathrm{ED}$, while $63.6 \%(\mathrm{n}=21)$ of those who presented a median symptomatology, and $57.1 \%(n=4)$ of those with severe symptoms, had severe ED $\left(\mathrm{X}^{2}(6)=14.9, \mathrm{p}\right.$ $=0.021) ;-$ among subjects who had stopped smoking from less than a year, none had severe or very severe COPD, while among those who had stopped smoking for more than 5 years, $83.3 \%(n=5)$ were suffering from severe COPD $\left(\mathrm{X}^{2}(12)=33.2, \mathrm{p}=0.001\right)$; - there was no case of severe or very severe COPD among patients with FVC in the normal range, while an equal distribution of patients with FVC was observed to be below the normality threshold among the different degrees of $\operatorname{COPD}\left(\mathrm{X}^{2}(4)=15.5, \mathrm{p}=0.004\right)$.

The Kruskal-Wallis test has revealed a weak connection between ED and age, while COPD seems to be associated with most of the spirometric parameters, BMI, quality of life perception and ED expressed as absolute score (Table 3 shows the medians and statistics of the test).

According to the Mann-Whitney test, there is no statistical difference between the characteristics of patients without ED, or at risk of COPD and those who had already developed the disease.

The degree of ED has been correlated in a statistically significant way with the severity levels of COPD $(\rho=-0.28$, $p=0.024)$ and age $(\rho=-0.33, p=0.007)$, or rather a more serious form of COPD and at an older age corresponds to a more important ED.

As expected, COPD seems related to many spirometric parameters and quality of life. In particular, more severe COPD corresponds to poorer values of spirometric parameters, but a better sense of well-being.

The results are reported in Table 4.

In order to complete the study, a more thorough analysis of the stratified correlation for the main characteristics of the patients has been performed both for COPD as well as for ED.

Based on the logistic regression analysis none of the monitored parameters emerged as a predictor of the presence of ED. Statistical evidence has led to the identification of forced expiatory volume corruption as the sole predictor of COPD. Specifically, it has been estimated that patients with FEV1 lower than $80 \%$ had about 5fold higher odds of presenting pathology than patients with normal parameters $(\mathrm{OR}=5.22, \mathrm{CI} 95=1.25,21.82$, $\mathrm{p}=0.023)$. 
Table 3.

Distribution of the variable medians associated with erectile dysfunction and COPD, for the different severity degrees of the disease, and Kruskal-Wallis test ( $\mathrm{X}^{2}, \mathrm{p}$-value).

\begin{tabular}{|cccccccccc}
\hline & & Absent (median) & Light (median) & Moderate (median) & Severe (median) & - & $X^{\mathbf{2}(3)}$ & $\mathbf{p}$-value \\
\hline ED categorical & Age & 61 & 61 & 67 & 66 & - & 7.98 & 0.046 \\
\hline & & & & & & & & \\
& & At risk (median) & Light (median) & Moderate (median) & Severe (median) & Very serious (median) & $X^{\mathbf{2}}$ (4) & $\mathbf{p}$-value \\
\hline COPD category & BMl & 27 & 29 & 25 & 31 & 23 & 13.0 & 0.011 \\
\hline & FV & 68 & 78 & 79 & 55 & 51 & 18.5 & 0.001 \\
\hline FEV1 & 68 & 54 & 63 & 41 & 25 & 29.7 & 0.000 \\
\hline IT & 63 & 52 & 61 & 53 & 40 & 12.1 & 0.017 \\
\hline FEF75\% & 39 & 44 & 29 & 16 & 10 & 27.4 & 0.000 \\
\hline PEF & 49 & 57 & 66 & 41 & 28 & 27.3 & 0.000 \\
\hline CV & 63 & 66 & 77 & 67 & 50 & 18.8 & 0.001 \\
\hline St.George & 97 & 100 & 93 & 73 & 74 & 15.8 & 0.003 \\
\hline DE score & 16 & 10 & 18 & 6 & 4 & 9.6 & 0.048 \\
\hline
\end{tabular}

Table 4.

Variables associated with erectile dysfunction and COPD, both categorical ( $\rho, p$-value).

\begin{tabular}{|c|c|c|c|}
\hline & Variables & $\rho$ & p-value \\
\hline \multirow[t]{2}{*}{ ED category $(n=66)$} & COPD category & $-0.278(*)$ & 0.024 \\
\hline & Age & $0.327(* *)$ & 0.007 \\
\hline \multirow[t]{7}{*}{ COPD category $(n=66)$} & 02 & $-0.244(*)$ & 0.048 \\
\hline & $\mathrm{FVC}$ & $-.359(* *)$ & 0.003 \\
\hline & FEV1 &. $.510(* *)$ & 0.000 \\
\hline & IT & $-.254(*)$ & 0.040 \\
\hline & FEF75\% & $-.490(* *)$ & 0.000 \\
\hline & PEF & $-.394(* *)$ & 0.001 \\
\hline & Quality of life (St. George) & $-.405(* *)$ & 0.001 \\
\hline
\end{tabular}

\section{Discussion}

On the basis of an analysis of correlation, the severity degree of ED and COPD appear to be associated: severe levels of COPD correspond to poorer erectile function (24). However, it was not possible to identify the predictors of ED through the analysis of logistic regression, although it appeared that both married patients as well as those with medium to severe urinary disorders suffered from a higher level of dysfunction. Furthermore, patients with mild disease or absence of disease had a lower mean age. The only predictive parameter of COPD was found to be forced expiatory volume lower than $80 \%$.

Many of the other spirometric parameters, although not predictory, resulted to be significantly correlated to a more severe form of COPD, as well as having a more positive perception of life quality. In addition to the previous results, the number of years as ex-smoker seems associated with the severity of COPD, or rather the greater part of those who had stopped smoking for more than 5 years had severe or very severe forms. Finally, according to the Kruskal-Wallis test, patients with varied degrees of pathology differ significantly in many of the spirometric parameters, for body mass index, for the perception of quality of life and for ED, expressed as a continuous variable. The need to study the pathologies on the basis of certain characteristics of patients, such as smoking habits, requires subdividing them into small samplesized groups.

This limits the efficacy of the study, as it does not allow generalization and the extension of the results to an entire population. A more appropriate study would provide for a stratified sample regarding both degrees of COPD severity, so as to obtain an equitable number of subjects suffering from the disease and at risk for COPD, as well as the different smoking habits.

At present, ED is increasingly establishing itself as the "sentinel symptom" of chronic diseases (hypertension, diabetes, ischemic heart disease): in this sense, it may paradoxically be a real "life saver" by which we can identify and eventually correct early phases of disease or hazardous lifestyles. It has also been emphasized how COPD is a "modifiable" condition before it becomes irreversible. Karadag (25) has shown how in patients with COPD, a high level of cytokines TNF- $\alpha$, the recognized marker of chronic inflammation, is in turn correlated with body weight, chronic hypoxemia and cigarette smoking. In the relationship between the two pathological conditions, moderate and severe levels of TNF- $\alpha$ are particularly high in ED, suggesting that even chronic inflammation may play a causative role in the onset of ED in patients with COPD (26), resulting in alterations in the vascular endothelium (27-30).

However, the high levels of plasma cytokines related to the degree of obesity in patients with ED had already been evaluated by Giuliano (31). COPD, as other diseases of the lung, can result in a condition, shared with the ED, of vasoconstriction and microvascular occlusion. In the lung, this leads to changes in the ventilation/perfusion ratio, intrapulmonary shunting, arterial hypoxemia and pulmonary hypertension, especially in the later stages of the disease. In both primary and secondary pulmonary hypertension, there is a decreased expression of e-NOS 
(endothelial nitric oxide synthase), pulmonary vascular endothelium.

The three aetiopathogenetic hypotheses are common in the two conditions: inflammatory, vascular and endocrine. Following sexual stimulation, non-adrenergic and non-cholinergic nerve endings (NANC) release NO, which through the second messenger, cGMP, induces the relaxation of the smooth muscles in the corpora cavernosa. The levels of cGMP are regulated by the action of PDE5, the enzyme responsible for the hydrolysis of cGMP in GMP. For the same functional cascade, this could also represent the rationale of treatment with PDE5 inhibitors, aimed at improving the ventilatory lung flow (32).

\section{Conclusions}

Both ED and COPD conditions share several risk factors and physiopathological mechanisms. These correlations can lead to some interesting assumptions of treatment, common to both diseases, although the importance of proper prevention, especially in younger patients, cannot be ignored.

ED and COPD may finally determine important ingravescence as well as often cause emotional, relational, social and sexual issues, with potentially depressive tendencies, not only in the patient but also, and especially, in the couple.

These conclusions confirm, once again, the need for a multi-speciality health policy for the prevention and management (33).

\section{REFERENCES}

1. Global Initiative for Chronic Obstructive Lung Diseases: Global strategy for the diagnosis, management and prevention of chronic obstructive pulmonary disease (COPD). Update January: 2, 2014.

2. NIH Consusus Development Conference Statement: Impotence. 1992; 10:1-31.

3. Feldman HA, Goldstein I, Hatzichristou DG, et al. Impotence and its medical and psychosocial correlates: results of the Massachusetts Male Aging Study. J Urol. 1994; 151:54-61.

4. Schouten BW, Bohnen AM, Dohle GR, Groeneveld FPM, et al. Risk factors for deterioration of erectile function: the Krimpen study. Int J Androl. 2007; 32:166-175.

5. Cazzola M, Bettoncelli G, Sessa E, et al. Prevalence of comorbidities in patients with chronic obstructive pulmonary disease. Respiration; international review of thoracic diseases 2010; 80:112-119

6. Gandaglia G, Briganti A, Jackson G, et al. A Systematic Review of the Association Between Erectile Dysfunction and Cardiovascular Disease. Eur Urol. 2014; 65:968-978.

7. Tamler R. Diabetes, obesity and erectile dysfunction. Gender Medicine 2009; Vol. 6 Theme Issue; 4-16.

8. Roumeguère TH, Wespes E, Carpentier Y, et al. Erectile dysfunction is associated with high prevalence of hyperlipidemia and coronary heart disease risk. Eur Urol. 2003; 44:355-359.

9. Benet AE, Melman A. The epidemiology of erectile dysfunction. Urol Clin North Am. 1995; 22:699-709.
10. Parazzini F, Menchini Fabris F, Bortolotti A, et al. on behalf of Gruppo Italiano Studio Deficit Erettile, Frequency and determinants of erectile dysfunction in Italy. Eur Urol. 2000; 37:43-49.

11. Gareri P, Castagna A, Francomano D, et al. Erectile dysfunction in the elderly: an old widespread issue with novel treatment perspectives. Int J Endocrinol. 2014; 2014; 873760.

12. Krane RJ, McKinlay JB. Impotence and its medical and psychological correlates: results of the Massachusetts Male Aging Study. J Urol. 1994; 151:54-61.

13. Giuliano F, Droupy S. Erectile dysfunction. Prog Urol. 2013; 23:629-637.

14. Tiengo A, Fadini GP, Avogaro A. The metabolic syndrome, diabetes and lung dysfunction. Diabetes Metab. 2008; 34:447-454.

15. Huang WJ, Chen KK, Chang LS. Correlation between voiding and erectile function in patients with symptomatic benign prostatic hyperplasia. J Clin Med Assoc. 2005; 68:178-182.

16. Braun M, Wassmer G, Klotz T, et al. Epidemiology of erectile dysfunction: results of the 'Cologne Male Survey'. Int J Impot Res. 2000; 12:305-311.

17. McInnes RA. Chronic illness and sexuality. Real Life. 2003; 179:263-266.

18. Lauretti S, Cardaci V. Broncopneumopatia cronica ostruttiva (BPCO) e disfunzione erettile (DE): implicazioni eziopatogenetiche multifattoriali. Urologia. 2008; 75:S11-S15.

19. Paap M, Bode C, Lenferink L, et al. Identifying key domains of health-related quality of life for patients with chronic obstructive pulmonary disease: the patient perspective. Health Qual Life Outcomes. 2014; 1:106-123.

20. Kaptein $A A$, van Klink RC, de Kok F, et al. Sexuality in patients with asthma and COPD. Respir Med. 2008; 102:198-204.

21. Collins EG, Halabi S, Langston M, et al. Sexual dysfunction in men with COPD: impact on quality of life and survival. Lung. 2012; 190:545-556.

22. Vijayan VK. Chronic obstructive pulmonary disease. Indian J Med Res. 2013; 137:251-269.

23. Fletcher EC, Martin RI. Sexual dysfunction and erectile impotence in chronic obstructive pulmonary disease. Chest. 1982; 81:413-421.

24. Koseoglu N, Koseoglu H, Ceylan E, et al. Erectile dysfunction prevalence and sexual function status in patients with chronic obstructive pulmonary disease. J Urol. 2005; 174:249-252.

25. Karadag F, Ozcan H, Karul AB, et al. Correlates of erectile dysfunction in moderate-to-severe chronic obstructive pulmonary disease patients. Respirology. 2007; 12:248-253.

26. Carneiro FS, Saiprazad Zemse B, et al. TNF- $\alpha$ infusion impairs corpora cavernosa reactivity. J Sex Med. 2009; 6 Suppl 3:311-319.

27. Aversa A. Strategies to improve endothelial function and its clinical relevance to erectile dysfunction. Eur Urol. Suppl. 2009; 8:71-79.

28. Rajfer J. Endothelial dysfunction as a cause of erectile dysfuction. Misdiagnosis or misnomer? Urology. 2004; 64:193-194.

29. Deanfild JE, Halcox JP, Rabelink TJ. Endothelial function and dysfunction: testing and clinical relevance. Circulation. 2007; 115:1285-1295.

30. Foresta C, Caretta N, Lana A, et al. Relationship between vascu- 
lar damage degrees and endothelial progenitor cells in patients with erectile dysfunction: effect of vardenafil administration and PDE5 expression in the bone marrow. Eur Urol. 2007; 51:1411-1417.

31. Giugliano F, Esposito K, Di Palo C, et al. Erectile dysfunction associates with endothelial dysfunction and raised pro-inflammatory cytokine levels in obese men. J Endocrinol Invest. 2004; 27:665-66.
32. Toque HA, Teixeira CE, Priviero FB, et al. Vardenafil, but not sildenafil or tadalafil, has calcium-channel blocking activity in rabbit isolated pulmonary artery and human washed platelets. Br. J. Pharmacol 2008; 154: 787-796.

33. Vincent EE, Singh SJ. Addressing the sexual health of patients with COPD: the needs of the patient and implications for health care professionals. Chronic Respiratory Disease. 2007; 4:111-115.

\section{Correspondence}

Stefano Lauretti, MD

stefanolauretti@gmail.com

Center of Sexual Medicine and Urological Rehabilitation. Day Surgery Unit, PSCR S. Caterina della Rosa, ASL Roma C, Rome, Italy

Vittorio Cardaci, MD

vittorio.cardaci@sanraffaele.it

Physiopathology and Respiratory Rehabilitation Unit IRCSS San Raffaele Pisana, Rome, Italy

Francesco Barrese, MD

fbarrese.urologo@gmail.com

Unit of Urology. Fabia Mater, Roma. Consultant in Urology IRCSS San Raffaele Rome, Italy

Luigino Calzetta, MD

luigicalz@gmail.com

Laboratory of Systems Approaches, IRCSS San Raffaele Pisana

Rome, Italy 